\title{
Control del avance del frente de llama en el lecho de sinterización de minerales de hierro ${ }^{(\bullet)}$
}

\author{
A. Cores*, J. Mochón*, I. Ruiz-Bustinza* y R. Parra**
}

\begin{abstract}
Resumen
En una planta piloto de sinterización se cargan, en la paila cúbica de $40 \mathrm{~cm}$ de lado, $110 \mathrm{~kg}$ de una mezcla de minerales de hierro, caliza y coque, donde se han introducido una serie de termopares a diferentes profundidades. Se realizan ensayos para estudiar la evolución del ancho de la zona de combustión y de la temperatura máxima del frente de llama a través del lecho de sinterización. Para el análisis de los resultados se utiliza un sistema de adquisición de datos formado por dos módulos conectados en serie, encargados de realizar la conversión analógico-digital. La entrada analógica es la salida de los termopares y la salida digital es la medida de la temperatura. Se dispone de un ordenador para la conservación y almacenamiento de los datos y para realizar interpolaciones que simulan el estado y evolución del frente de llama a través del lecho.
\end{abstract}

Palabras clave Sinterización; Frente de llama; Combustión; Minerales de hierro; Sensores de temperatura; Ajuste de algoritmos; Sistema de adquisición de datos.

\section{Control of the flame front advance in a sintering bed of iron ores}

Abstract

Keywords

\begin{abstract}
A sintering pan of $40 \mathrm{~cm}$ cubed is loaded with a mixture of iron ores, limestone and coke weighing $110 \mathrm{~kg}$ in a sintering pilot plant. In this sintering pan, a series of thermocouples have been introduced at different depths. Tests have been carried out to study the width of the combustion zone and the maximum temperature of the flame front across the sintering bed. For the analysis of the results, a data acquisition system was used. This consisted of two modules connected in serie, for performing the analogue-digital conversion. The analogue entry point is the exit point of the thermocouples and the digital exit point was the temperature average. A computer was used for conserving and storing the data and for carrying out interpolations, simulating the state and evolution of the flame front across the bed.
\end{abstract}

Sintering; Flame front; Combustion; Iron ores; Temperature sensors; Algorithmic adjustments; Data acquisition system.

\section{INTRODUCCION}

El proceso de sinterización se utiliza para aglomerar una mezcla de minerales de hierro, fundentes y coque, de tamaño de partícula inferior a $8 \mathrm{~mm}$, de tal forma que el sinterizado producido, con tamaño tamizado entre 12 y $35 \mathrm{~mm}$, una vez cargado en el horno alto pueda soportar las presiones y temperaturas del horno.Para sinterizar, la mezcla mineral se somete previamente a una granulación, que consiste en homogenizar la mezcla en un tambor giratorio, con la adición de un 6-8 \% de agua, durante unos minutos.
Los gránulos formados se cargan sobre la parrilla de la banda de sinterización, donde la temperatura de los gránulos se eleva entre 1.200 y $1.350^{\circ} \mathrm{C}$ para producir la fusión parcial y producir material semifundido, el cual, durante el enfriamiento posterior, cristaliza en varias fases minerales de distinta composición química y morfológica. Estas fases son: hematita no asimilada o residual, también denominada hematita primaria; hematita precipitada o hematita secundaria; magnetita; silicoferritos de calcio y aluminio (SFCA) y ganga formada, principalmente, por silicatos de calcio, hierro y magnesio ${ }^{[1-5]}$.

(•) Trabajo recibido el día 29 de Septiembre de 2009 y aceptado en su forma final el día 8 de Febrero de 2010.

* Centro Nacional de Investigaciones Metalúrgicas, CENIM (CSIC). Avda. Gregorio del Amo 8, 28040 Madrid, España. alcores@cenim.csic.es; jmochon@cenim.csic.es;irbustinza@cenim.csic.es.

** Dpto. Ingeniería Metalúrgica, Universidad de Concepción Edmundo Larenas, 285 Concepción, Chile. rparra@udec.cl. 
La energía del proceso es suministrada por la combustión del coque, en la parte superior del lecho de sinterización, y el aire, que es succionado a través del lecho, desplaza la zona de combustión (frente de llama) hacia abajo y, de esta forma, se realizan las reacciones de sinterización. La composición de las fases y la calidad del sínter están muy influenciadas por la temperatura de la zona de combustión, por la distribución de calor en el lecho y por la formación del fundido.

La figura 1 muestra la distribución ideal de calor, en el lecho. El tiempo de elevación de temperatura en la zona de alta temperatura (superior a $1.100{ }^{\circ} \mathrm{C}$ ), debe ser corto (1,5 $\mathrm{min}$ ) debido a que, en esta zona, la presión parcial del oxígeno $\mathrm{Po}_{2}$ es baja debido a la combustión del coque y se forma, fácilmente, $\mathrm{FeO}$, lo cual perjudica la reductibilidad del sínter. El tiempo de descenso de temperatura (hasta $1.100^{\circ} \mathrm{C}$ ) debe ser largo (de 3 a $5 \mathrm{~min}$ ) para obtener una buena resistencia, por la formación de una matriz de ganga en la estructura del sinterizado, que se favorece por la presencia de $\mathrm{SiO}_{2}$.

Los perfiles tiempo-temperatura (Fig. 1) han sido medidos en varias posiciones del lecho. Los perfiles indican que según la zona de combustión desciende a través del lecho, el ancho de esta zona y la Tmax aumentan. Para conseguir una Tmax uniforme, en algunas plantas se realiza la sinterización en doble capa, que consiste en la preparación del lecho con

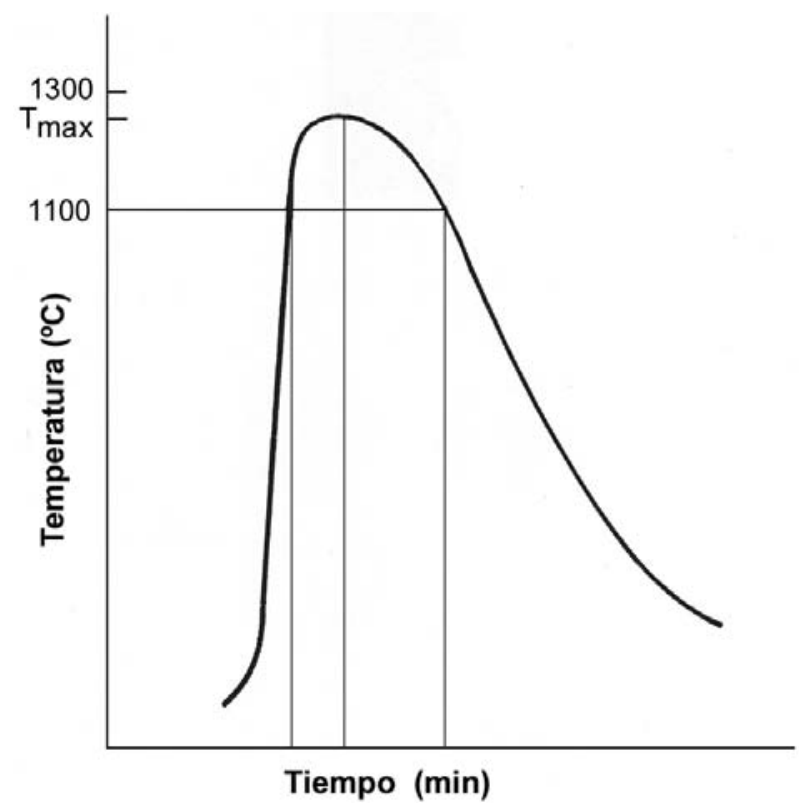

Figura 1. Perfil de la distribución ideal del frente de llama.

Figure 1. Ideal distribution profile of the flame front. un contenido en coque más elevado en la capa superior que en la capa inferior, para contrarrestar la tendencia de la Tmax a elevarse ${ }^{[6-8]}$.

Además de la doble capa, para el control de la distribución de calor en el lecho se ha desarrollado un equipo de medida continua que señala, directamente, la distribución de las temperaturas en el lecho y un dispositivo para la medida de la distribución del volumen aspirado a través de la paila de sinterización ${ }^{[9}$ y 10]. La distribución del calor se puede controlar por medio de la velocidad de la paila y el contenido de coque en la mezcla.

Se han realizado ensayos en un horno de calentamiento rápido que permiten construir un perfil térmico de la muestra (hematita + caliza), que simula el perfil de la planta de sinterización ${ }^{[11}$ y 12$]$.

Se ha estudiado la sinterización con la ayuda de modelos de simulación ${ }^{[12]}$ y se ha encontrado una buena correlación entre la distribución de calor experimental y la calculada por el modelo. En otro trabajo $^{[10]}$, se ha formulado una ecuación para la distribución de calor, con una buena correlación entre los datos experimentales y los calculados.

En una planta piloto de sinterización ha sido posible conseguir una distribución térmica uniforme a través de la dirección transversal del lecho de sinterización, por medio del control automático del nivel de carga, usando un medidor de nivel ultrasónico que relaciona la temperatura del gas debajo de las cajas de viento con el nivel de carga ${ }^{[13]}$. Se han conseguido mejoras en la eficiencia, productividad y calidad del sínter.

En dos bandas de sinterización se ha desarrollado un modelo que combina el balance térmico con el análisis de $\mathrm{CO}, \mathrm{CO}_{2}$ y $\mathrm{O}_{2}$ en una caja de viento, que permite la elaboración de un sistema de control de la banda ${ }^{[14]}$. Así, es posible determinar la posición del frente de llama, la Tmax y la cantidad de líquido (fundido) formado en la zona de combustión.

Se ha propuesto un modelo de la combustión y transferencia de calor en un lecho de sinterización, en una investigación realizada en laboratorio. Los datos calculados por el modelo, de los perfiles de las curvas correspondientes a la propagación del frente de llama, de la composición de los gases de combustión $\left(\mathrm{CO}, \mathrm{CO}_{2}\right.$ y $\left.\mathrm{O}_{2}\right)$ y del contenido de coque en la mezcla mineral, se comparan con los datos experimentales de la paila y se observa una buena correlación ${ }^{[15]}$.

En otra investigación se ha propuesto un modelo para la velocidad de propagación del frente de llama a través del lecho. Los parámetros principales de la investigación son la combustión del coque y el flujo y contenido de $\mathrm{CO}_{\text {y }} \mathrm{CO}_{2}$ en el gas que atraviesa el lecho. Hay una buena correlación entre los datos 
obtenidos por el modelo y los resultados experimentales de la planta piloto ${ }^{[16]}$.

En el presente trabajo se fabrica, en una planta piloto de sinterización, una serie de sinterizados a partir de una misma mezcla mineral. En cada ensayo se introduce en el lecho de sinterización una serie de termopares, a profundidades diferentes, que registran las temperaturas en el lecho durante el ensayo. Mediante un sistema de adquisición de datos se puede estimar, el estado y la evolución del frente de llama y el ancho de la zona de combustión a través del lecho.

\section{RELACIÓN ENTRE EL FRENTE DE LLAMA Y LA ESTRUCTURA DEL SINTERIZADO}

Cuando la sinterización se realiza a temperatura inferior a $1.300^{\circ} \mathrm{C}$, en el lecho de sinterización se genera, a unos $1.200^{\circ} \mathrm{C}$, un fundido (que consiste, principalmente, en $\mathrm{Fe}_{2} \mathrm{O}_{3}$ y $\mathrm{CaO}$ ) y el óxido de hierro y las partículas minerales finas se asimilan en el fundido. Si el fundido penetra en el grano de hematita, se produce una rotura interfacial quedando una hematita primaria ( $\sin$ fundir) que se considera beneficiosa para el sinterizado porque mejora el índice de reductibilidad (RI). Cuando el $\mathrm{CaO}$ y el $\mathrm{Al}_{2} \mathrm{O}_{3}$ se asimilan en el fundido, éste reacciona con el óxido de hierro y genera ferrito cálcico acicular (de tamaño inferior a $10 \mu \mathrm{m}$ ) conteniendo $\mathrm{Al}_{2} \mathrm{O}_{3}$ y $\mathrm{SiO}_{2}$ como disoluciones sólidas. Estos silicoferritos de calcio y aluminio (SFCA) se consideran componentes muy beneficiosos para la estructura del sinterizado, puesto que poseen buena reductibilidad y aportan resistencia mecánica al sinterizado mejorando el índice shatter (S I). Al sinterizar a baja temperatura disminuye la formación de magnetita (menor $\mathrm{FeO}$ ) y el sinterizado mejora el RI y mejora (disminuye) el índice de la degradación con la reducción $(\mathrm{RDI})^{[7,11 \text { y 17]. }}$

Cuando se sinteriza a temperatura superior a $1.300{ }^{\circ} \mathrm{C}$, parte del ferrito se disuelve y funde para convertirse en hematita o magnetita y en componentes de ganga ${ }^{[18-20]}$. Al enfriarse el fundido se forman, como fases nuevas, grandes cristales de ferrito que se reducen peor que el ferrito acicular, y hematita secundaria que es perjudicial para el RDI ${ }^{[17]}$.

En la figura 2 se ofrece un esquema del desarrollo de las diferentes estructuras en función de la Tmax alcanzada en el lecho.

En la tabla I se puede apreciar cómo varían la composición de las fases y los índices de calidad del sinterizado en función de la Tmax alcanzada en el lecho ${ }^{[5]}$. Se observa que los mejores resultados se obtienen en el intervalo de $1.225-1.275^{\circ} \mathrm{C}$, con un

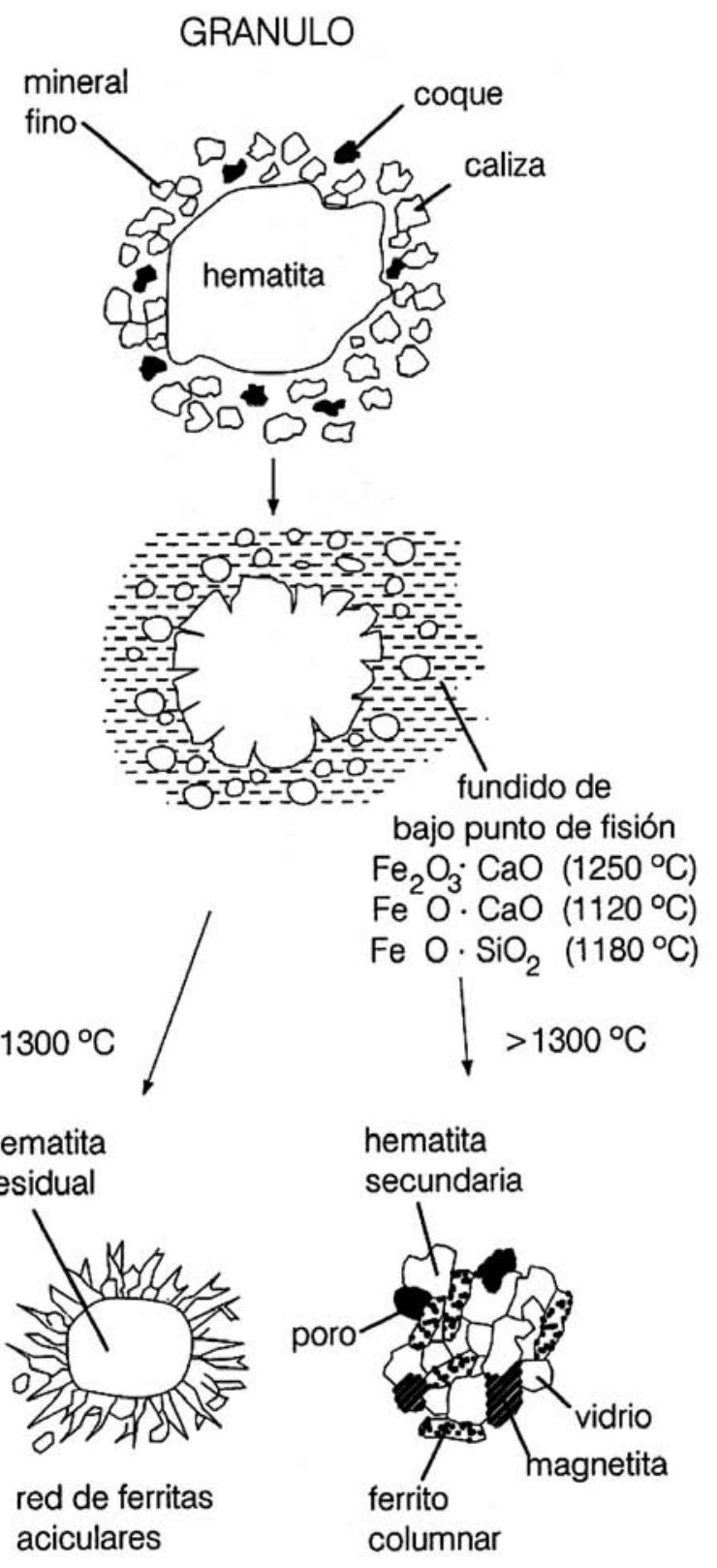

Figura 2. Desarrollo de la estructura del sinterizado.

Figure 2. Development of sintering texture.

porcentaje máximo de ferritos, alto de hematita primaria, bajo de hematita secundaria, buena porosidad y buenos índices de calidad (FeO, RDI, RI y SI).

\section{EXPERIMENTAL}

\subsection{Planta piloto de sinterización}

La parte experimental se ha realizado en la planta piloto de sinterización del CENIM, que dispone de 
Tabla I. Valores aproximados de la composición de las fases y calidad de los sinterizados en función de la temperatura máxima del lecho, \%

Table I. Approximate values of phase and sinter quality versus maximum temperature in the bed, \%

$\operatorname{Tmax}{ }^{\circ} \mathrm{C}$

$1.175-1.225 \quad 1.225-1.275 \quad 1.275-1.350$

\begin{tabular}{lrrc}
$\begin{array}{l}\text { Hematita } \\
\text { primaria } \\
\text { Hematita }\end{array}$ & 50 & 42 & 22 \\
$\quad$ secundaria & 5 & 5 & 20 \\
Magnetita & 10 & 15 & 20 \\
SFCA & 35 & 38 & 30 \\
Vidrio + & & & \\
$\quad 2 \mathrm{CaO} \cdot \mathrm{SiO}_{2}$ & 7 & 10 & 12 \\
Porosidad & 35 & 30 & 15 \\
$\mathrm{FeO}$ & 3 & 4 & 5,5 \\
$\mathrm{RDI}$ & 30 & 32 & 36 \\
$\mathrm{RI}$ & 72 & 70 & 64 \\
$\mathrm{SI}$ & 93 & 94 & 95 \\
\hline
\end{tabular}

una paila de 40x40 cm de sección transversal y 40 cm de altura, y está equipada con un circuito de succión capaz de crear depresiones superiores a 1.470 $\mathrm{MPa}$, un sistema de ignición de propano y un sistema para la medida del volumen de gas de entrada y la temperatura del gas de salida. El tiempo de la sinterización es próximo a $20 \mathrm{~min}$.

En la figura 3 se ofrece el dispositivo experimental utilizado. La paila se carga con $110 \mathrm{~kg}$ de mezcla mineral y, en la carga, se introduce una serie de termopares, en posiciones distintas, que están conectados al sistema de captación de datos.

\subsection{Composición de la mezcla mineral}

En la tabla II se ofrece la composición de la mezcla mineral utilizada para cada ensayo, formada por cinco minerales de hierro, finos de retorno de sínter y caliza, como fundente. Los finos de retorno se obtienen de otro sínter ya fabricado ${ }^{[21]}$.

El coque se añade a la mezcla mineral, en un 3,3 \%. El coque se analizó en el Instituto Nacional del Carbón de Oviedo (INCAR), con el resultado siguiente: $83,50 \%$ carbono, $12,00 \%$ cenizas, $2,20 \%$ materia volátil, 0,95 \% humedad, 0,25\% hidrógeno,

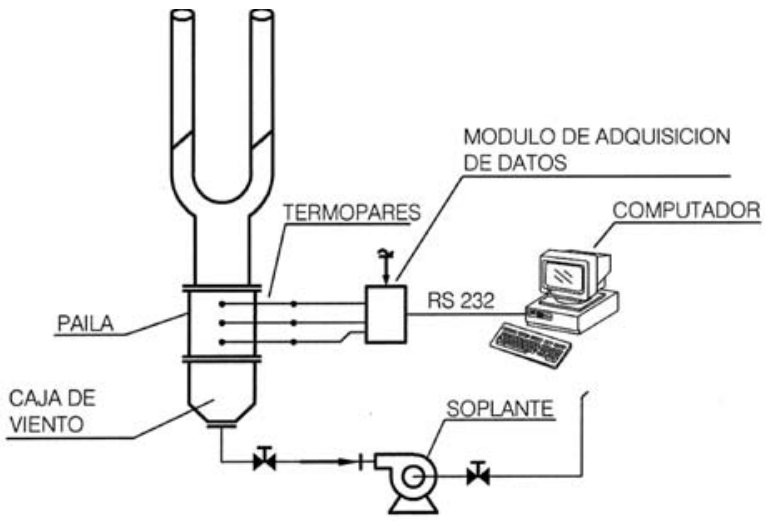

Figura 3. Sistema experimental de adquisición de datos durante los ensayos en la planta piloto de sinterización.

Figure 3. Experimental system of data acquisition during the tests in the sintering pilot plant.

0,96 \% nitrógeno, 0,10 \% azufre y un índice de reactividad CRI de 60,46.

\subsection{Realización de los ensayos de sinterización}

En este trabajo se han fabricado sinterizados a partir de la misma mezcla mineral (Tabla II). La mezcla se homogeniza en un mezclador, con la adición de un $7 \%$ de agua. A continuación, se carga la mezcla en la paila y se colocan los termopares.

Los termopares deben medir la temperatura de la carga durante el período de combustión y se ha procurado que el efecto del entorno exterior sea mínimo y, para ello, se instalaron en el tercio central de la paila y de la forma más centrada posible. Se introducen los termopares en la carga, en 8 alturas para tener 8 puntos de control para el avance del frente de llama.

Se han realizado los ensayos en una carga de composición química constante, con las mismas condiciones de quemado, y se ha observado cómo fluctúan los parámetros físico-químicos y térmicos. Esto, permite definir unos valores de los intervalos de credibilidad de los parámetros y acotar cuáles de las variaciones se deben al proceso y cuáles pertenecen a la variabilidad intrínseca de los materiales y de las variables.

Por último se ha procedido a estudiar el cambio de algunas variables en el seno de la carga de sinterización. Sobre todo, se ha intentado ver cómo varían algunas propiedades con la profundidad del lecho o, lo que es lo mismo, con la temperatura máxima o con la cantidad de calor disponible para la sinterización. 
Tabla II. Composición de la mezcla mineral, \% en masa

Table II. Composition of the mineral mix, \%mass

\begin{tabular}{lcccccccccc}
\hline & $\begin{array}{c}\text { \% en la } \\
\text { mezcla }\end{array}$ & $\begin{array}{c}\mathrm{Fe} \\
\text { total }\end{array}$ & $\mathbf{C a O}$ & $\mathbf{M g O}$ & $\mathbf{A l}_{\mathbf{2}} \mathbf{O}_{\mathbf{3}}$ & $\mathbf{S i O}_{\mathbf{2}}$ & $\mathbf{M n O}$ & $\mathbf{N a}_{\mathbf{2}} \mathbf{O}$ & $\mathbf{K}_{\mathbf{2}} \mathbf{O}$ & $\mathbf{P C}$ \\
\hline Cercal & 15,3 & 49,5 & 0,18 & 0,30 & 1,66 & 16,54 & 0,11 & 0,014 & 0,029 & 9,37 \\
Nimco & 15,3 & 68,8 & 0,12 & 0,15 & 1,02 & 4,58 & 0,12 & 0,013 & 0,032 & 1,80 \\
Dempo & 15,3 & 66,0 & 0,14 & 0,22 & 1,38 & 4,20 & 0,07 & 0,013 & 0,024 & 2,57 \\
Mount Wright & 11,9 & 64,3 & 0,18 & 0,20 & 0,42 & 4,34 & 0,04 & 0,011 & 0,017 & 1,01 \\
Tazadit & 18,5 & 63,7 & 0,13 & 0,02 & 0,90 & 7,55 & 0,02 & 0,017 & 0,023 & 1,10 \\
Finos de retorno & 16,9 & 55,0 & 10,5 & 2,08 & 1,23 & 5,50 & 0,28 & 0,024 & 0,039 & 0,42 \\
Caliza & 6,8 & 0,48 & 54,4 & 0,55 & $<0,5$ & 1,35 & 0,02 & 0,018 & 0,025 & 41,9 \\
\hline
\end{tabular}

$\mathrm{PC}=$ pérdidas por calcinación

\subsection{Sensores}

El sensor está formado por el termopar y la vaina protectora. Dado que las temperaturas de sinterización están en el intervalo de $1.150-1.350^{\circ} \mathrm{C}$, se seleccionó el termopar, tipo S, de polo positivo $\mathrm{Pt} 10 \% \mathrm{Rh}$, polo negativo $\mathrm{Pt}$ y rango de temperaturas de $-50 \mathrm{a}$ $1.768^{\circ} \mathrm{C}$.

Se ha utilizado una vaina porosa protectora, con un $90 \%$ de alúmina, porosidad abierta del $27 \%$, conductividad térmica $3 \mathrm{~W} / \mathrm{m}^{\circ} \mathrm{C}$, densidad $2,5 \mathrm{~g} / \mathrm{cm}^{3}$, límite de aplicación $1.600^{\circ} \mathrm{C}$ y buena resistencia al choque térmico.

Con esta vaina, el termopar medirá la temperatura de la vaina y no la temperatura de combustión, debido a la inercia térmica del material de la vaina. Debido a la buena calidad de la vaina la inercia térmica es pequeña. Esto es importante debido a que el proceso a medir es muy rápido.

\subsection{Sistema de adquisición de datos}

Este sistema está formado por dos módulos conectados en serie encargados de realizar la conversión analógico-digital. La entrada analógica es la salida de los termopares y la salida digital es la medida de la temperatura. La ventaja de este sistema es que los módulos se encuentran montados en una caja metálica y aparte del ordenador personal que sirve de almacenamiento y que se comunica con el sistema mediante un cable RS-232. Esto ocasiona que la señal que viaja sea una señal digital y, por tanto, menos influenciada por el entorno ya que reduce el número de cables y conexiones.

Al final del proceso, se encuentra el ordenador personal, encargado de la conservación y almacenamiento de los datos así como de las interpolaciones para simular el estado y la evolución del frente de llama.

El almacenamiento se lleva a cabo por un software específico de los módulos que componen el módulo SAD, que crea ficheros cuyo nombre es la fecha del ensayo y que se guarda en formato ASCII, con la información siguiente:

- Módulo al que pertenece.

- Número de canal.

- Tiempo de medida.

- Temperatura instantánea.

Las medidas se han programado para que se efectúen cada segundo y no se proceda a su integración.

\section{RESULTADOS Y DISCUSIÓN}

En una planta piloto de sinterización se ha realizado una serie de ensayos iniciales, probando 2 tipos de termopares y 3 tipos de vainas, que ha permitido elegir el mejor termopar y vaina. Se ha determinado la mejor colocación de los termopares en el lecho para los ensayos que se indican a continuación.

\subsection{Ensayos realizados}

\subsubsection{Ensayo 1}

Durante la sinterización, se colocan 15 termopares en unas posiciones que serán estándar para todos los ensayos, de la siguiente forma: 
- 14, en el interior de la paila situados a alturas de $4,8,5,13,18,5,22,26,5,31$ y $36 \mathrm{~cm}$.

- 1 , en el conducto de humos.

El efecto básico de este ensayo es el desfase, en tiempo, entre la temperatura medida y la real del medio. La medida registrada lleva cierto retraso respecto a la real. El intervalo de medida es de $1 \mathrm{~s}$.

En la figura 4 se observa la evolución térmica del frente de llama a lo largo del lecho y se puede apreciar cómo aumenta el ancho de la zona de combustión y la temperatura máxima del frente de llama, a medida que el frente avanza a través del lecho, lo cual coincide con otras investigaciones realizadas ${ }^{[6-8]}$. Los datos de la figura 4 permiten establecer el valor medio de la Tmax del ensayo en $1.288^{\circ} \mathrm{C}$ (Tabla III).

\subsubsection{Ensayo 2}

En este ensayo y en los siguientes se intenta reproducir el mismo esquema que el ensayo 1 . De esta forma, se procede a instalar los termopares en los mismos lugares y a las mismas alturas.

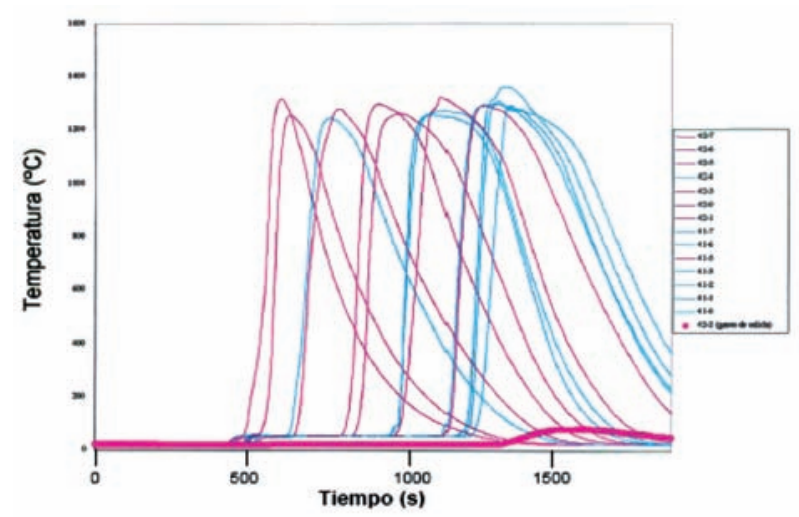

Figura 4. Evolución térmica del frente de llama (ensayo 1).

Figure 4. Thermic evolution of the flame front (test 1).

Tabla III. Valor medio de la temperatura máxima medida en cada ensayo

Table III. Average maximum temperature measured in each test

\begin{tabular}{ccccccc}
\hline Ensayo & $\mathbf{1}$ & $\mathbf{2}$ & $\mathbf{3}$ & $\mathbf{4}$ & $\mathbf{5}$ & $\mathbf{6}$ \\
\hline $\operatorname{Tmax},{ }^{\circ} \mathrm{C}$ & 1.288 & 1.264 & 1.243 & 1.216 & 1.192 & 1.221
\end{tabular}

Las curvas de la evolución térmica se muestran en la figura 5.

\subsubsection{Ensayo 3}

En la figura 6 a) se muestra la evolución térmica que corresponde a este ensayo y, en la figura $6 \mathrm{~b}$ ) se puede observar el frente de llama reconstruido.

\subsubsection{Ensayos 4, 5 y 6}

Las curvas de la evolución térmica de estos ensayos se ofrecen en las figuras 7, 8 y 9, respectivamente. Se puede observar la tendencia generalizada del aumento del ancho de la zona de combustión y del aumento de la Tmax, a medida que el frente de llama avanza a través del lecho.

\subsection{Análisis de las características de los sinterizados}

En la tabla IV se ofrece el análisis químico del sinterizado fabricado en el ensayo 6. El valor del índice RDI es $27 \%$.

En el ensayo 6 se tomaron varias muestras del sinterizado, a diferentes niveles del lecho, para ver la variabilidad de los valores del RDI y del contenido de $\mathrm{FeO}$ en el seno de la carga sinterizada. Los resultados se muestran en la figura 10.

Se puede observar cómo aumenta el valor del RDI con la profundidad del lecho. Esto, se debe a que a

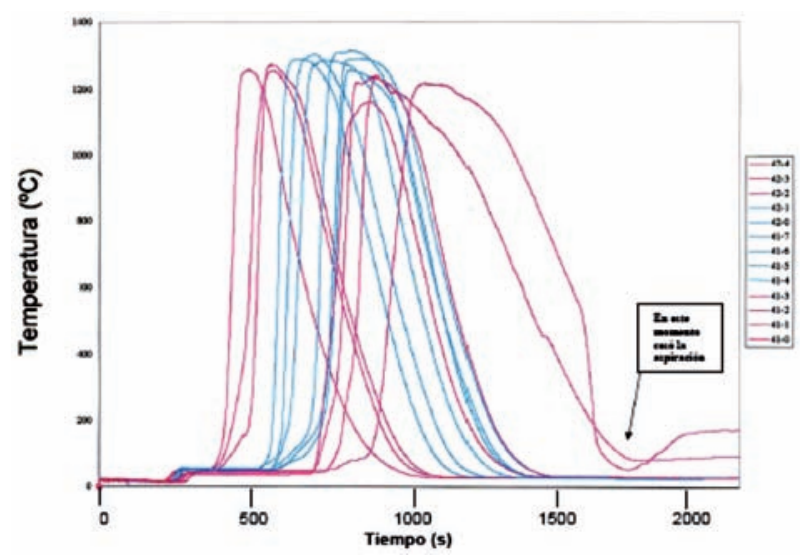

Figura 5. Evolución térmica del frente de llama (ensayo 2).

Figure 5. Thermic evolution of the flame front (test 2). 

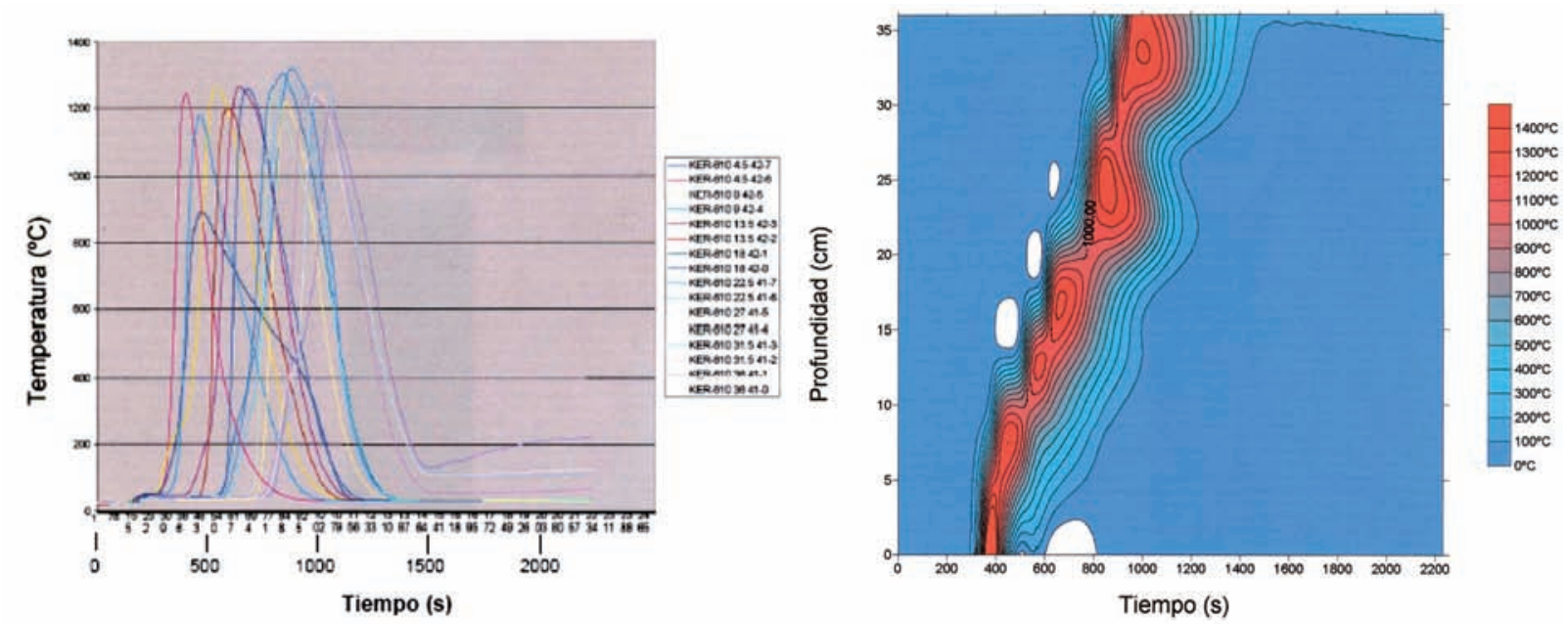

Figura 6. a) Evolución térmica del frente de llama (ensayo 3); b) Frente de llama reconstruido.

Figure 6. a)Thermic evolution of the flame front (test 3); b) Flame front reconstructed.

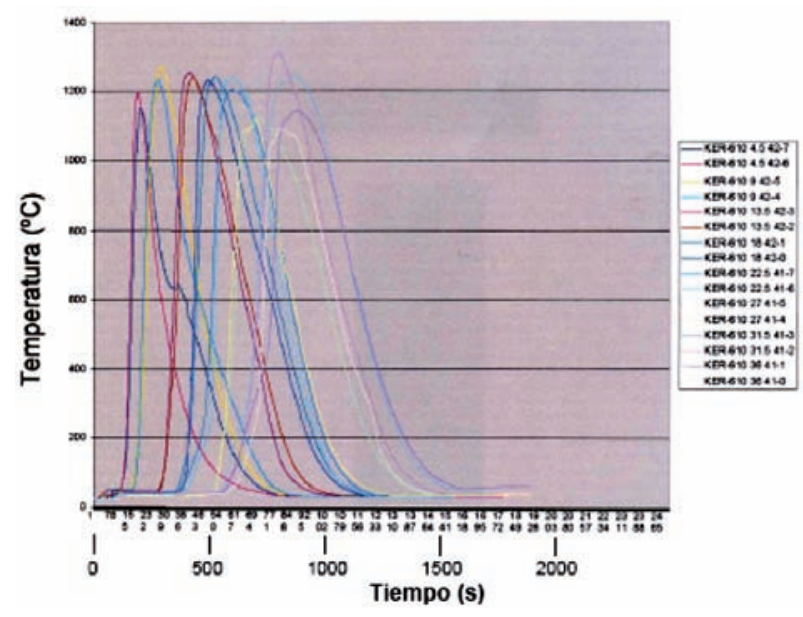

Figura 7. Evolución térmica del frente de llama (ensayo 4).

Figure 7. Thermic evolution of the flame front (test 4).

medida que el frente de llama avanza a través del lecho, también aumenta la Tmax y, con ello, aumenta el contenido de hematita secundaria y esto ocasiona un aumento en el valor del RDI, lo cual es perjudicial para la calidad del sínter ${ }^{[17]}$.

También, se observa un ligero aumento en el contenido de $\mathrm{FeO}$ a través del lecho, debido a que con el aumento de la Tmax disminuye el contenido de hematita primaria, aumenta el contenido de magnetita y, por tanto, aumenta el $\mathrm{FeO}$, lo que ocasiona una disminución en la reductibilidad del sínter ${ }^{[5]}$.

En la figura 11 se ofrece un aspecto de la microestructura de una muestra de sinterizado fabricado en el ensayo 6, realizada en un microscopio electrónico de barrido con microsonda JEOL, modelo JSM-840, con un analizador LINK AN-10000. Se aprecia la presencia de fases muy entremezcladas, con la estructura favorable formada por núcleos de hematita rodeados de ferritos aciculares.

\subsection{Análisis de los resultados térmicos}

\subsubsection{Evolución de la temperatura máxima del frente de llama con la profundidad del lecho}

Se ha comprobado la tendencia del valor de la Tmax del frente de llama en cada punto del lecho, utilizando los valores de los ensayos realizados. El resultado se ofrece en la figura 12 . Se puede apreciar cómo aumenta la Tmax con la profundidad del lecho.

En la figura 13 se muestra el análisis estadístico de los valores de la Tmax, con un valor medio de $1.223^{\circ} \mathrm{C}$ y una desviación típica de 94 para 71 casos.

\subsubsection{Evolución del ancho de la zona de sinterización con la profundidad del lecho}

Se entiende por ancho de la zona de sinterización al intervalo de tiempo durante el cual los valores de la temperatura del lecho se hallan por encima de los $900^{\circ} \mathrm{C}$. Estos valores se relacionan con los valores 


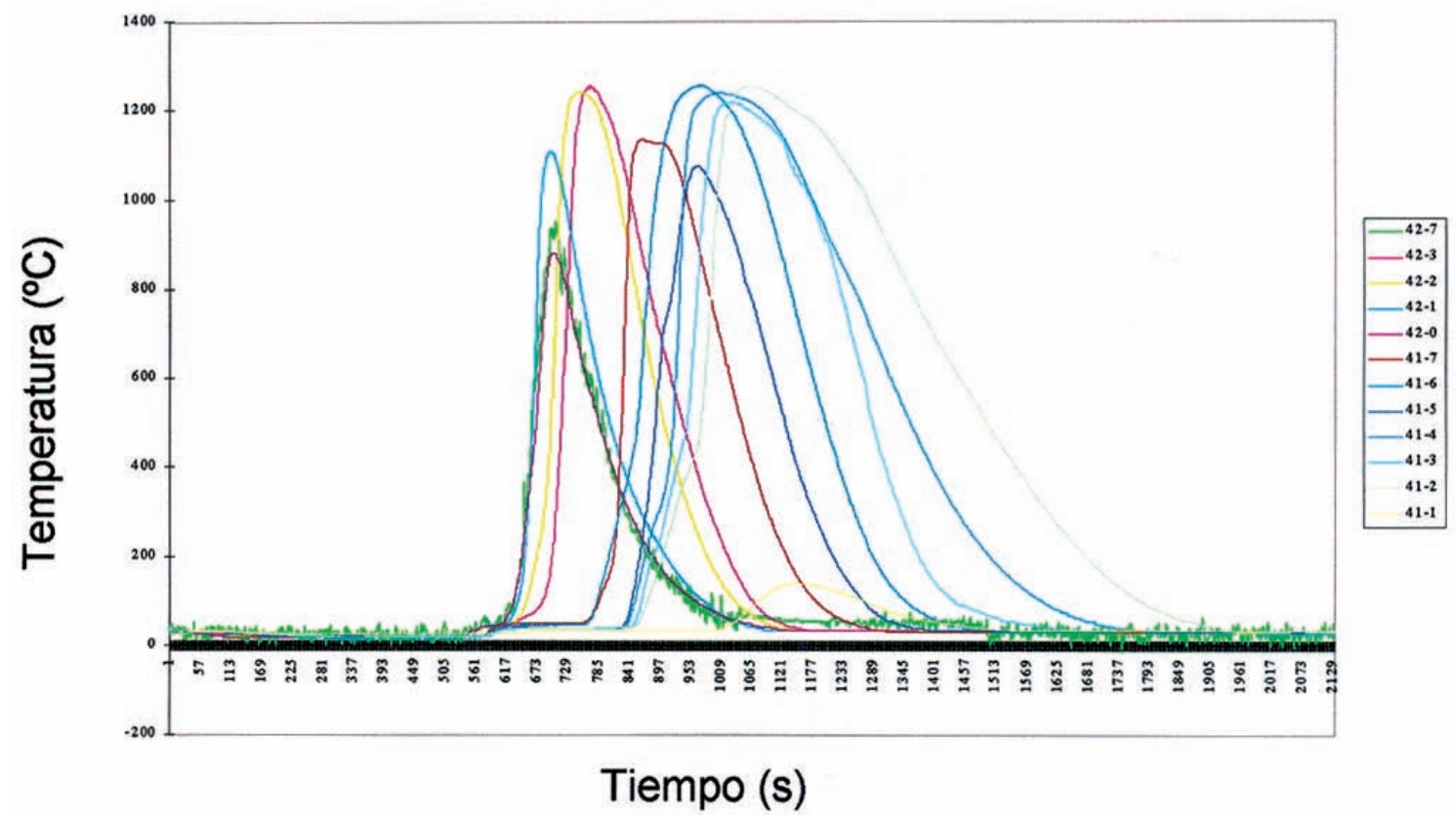

Figura 8. Evolución térmica del frente de llama (ensayo 5).

Figure 8. Thermic evolution of the flame front (test 5).

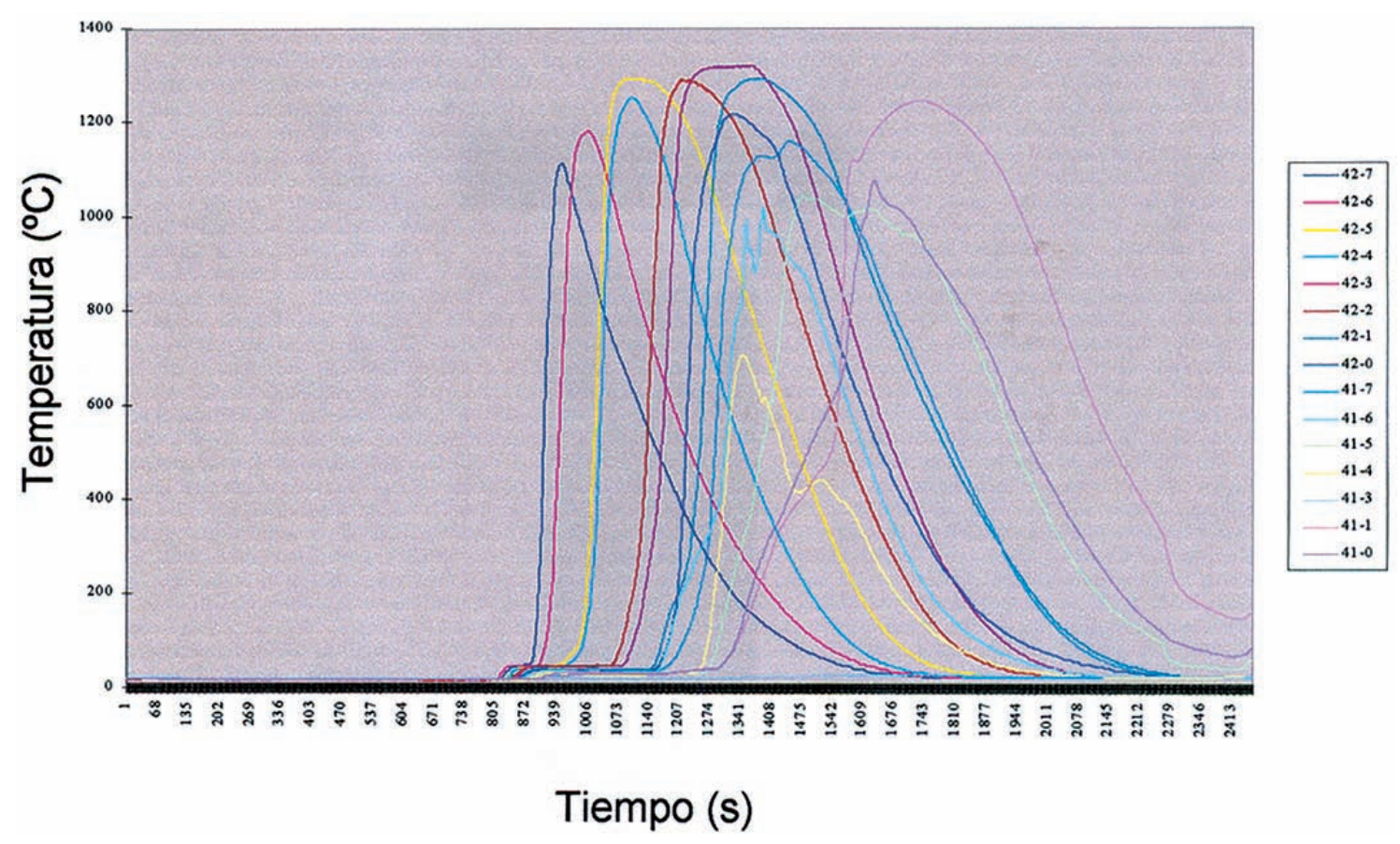

Figura 9. Evolución térmica del frente de llama (ensayo 6).

Figure 9. Thermic evolution of the flame front (test 6).

de la profundidad del lecho, como se muestra en la figura 14.

Se puede apreciar como aumenta este ancho con la profundidad, debido a que, en las capas superiores del lecho, los materiales están más cerca de la succión, el aire aspirado entra frío en el lecho y las condiciones de enfriamiento son más rápidas, por lo que la banda es menor. En las capas inferiores, el material 
Tabla IV. Análisis químico del sinterizado fabricado en el ensayo $6, \%$ en masa

Tabla IV. Chemical analysis of the sintering made in the test $6, \%$ mass

\begin{tabular}{lc}
\hline \multicolumn{1}{c}{ Elemento } & $\%$ \\
\hline Fe total & 55,6 \\
$\mathrm{FeO}$ & 4,7 \\
$\mathrm{CaO}$ & 11,1 \\
$\mathrm{MgO}$ & 1,90 \\
$\mathrm{Al}_{2} \mathrm{O}_{3}$ & 1,44 \\
$\mathrm{SiO}^{2}$ & 5,25 \\
$\mathrm{MnO}_{\mathrm{Na}} \mathrm{O}$ & 0,90 \\
$\mathrm{~K}_{2} \mathrm{O}$ & 0,021 \\
$\mathrm{P}$ & 0,033 \\
$\mathrm{~S}$ & 0,047 \\
\hline
\end{tabular}

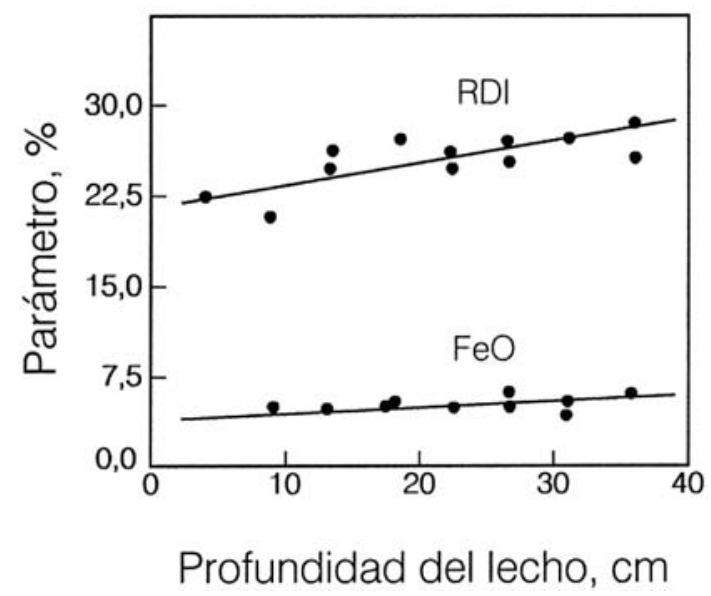

Figura 10. Índices de calidad del sinterizado fabricado en el ensayo 6 .

Figure 10. Quality index of the sintering made in the test 6.

está caliente por la propia combustión y, por tanto, este calor es transmitido al aire que pasa a su través. Este aire caliente no enfría la carga con tanta eficiencia y el ancho de la zona de sinterización es mayor. El valor medio de este ancho es de 204 s, con una desviación típica de 78 para 54 casos, según se observa en la figura 15.

En algunos ensayos se ha observado una anomalía térmica entre los 25 y $30 \mathrm{~cm}$ de profundidad, debida aun calentamiento muy rápido, adelantando,

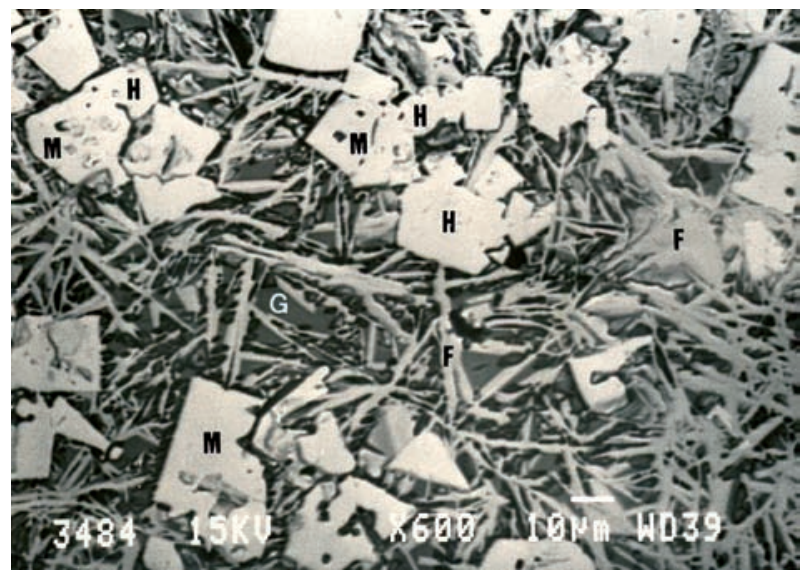

Figura 11. Microestructura de una muestra de sinterizado fabricado en el ensayo 6 , con presencia de fases muy entremezcladas (x600). $\mathrm{H}$ = hematita; $\mathrm{M}=$ magnetita; $\mathrm{F}=$ red de ferritos aciculares y columnares; $\mathrm{G}=$ ganga. Presencia de poros (negro).

Figure 11. Microestructure of a sintering sample made in the test 6 , with presence of very intermingle phases (x600). $H=$ hematite; $M=$ magnetite; $F=$ Lattice of acicular and columnar ferrites; $G$ = gange. Presence of pores (black).

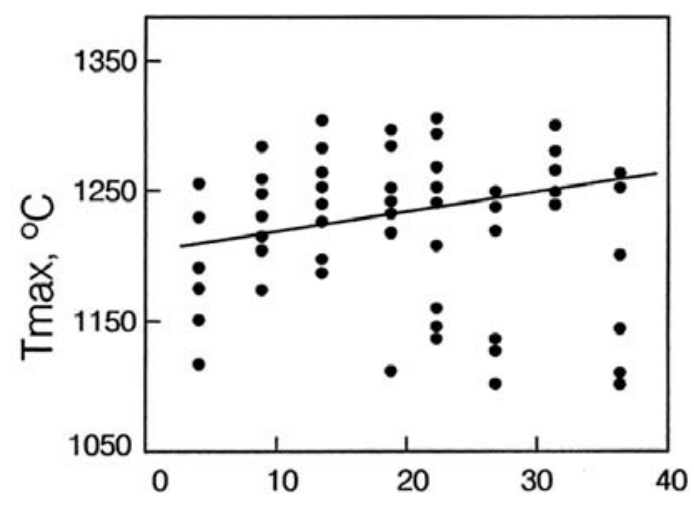

Profundidad del lecho, $\mathrm{cm}$

Figura 12. Evolución de la temperatura máxima con la profundidad del lecho.

Figure 12. Maxim temperature evolution with the depth bed.

en ocasiones, al calentamiento que se produce a 36 $\mathrm{cm}$. También, se ha observado que la Tmax no es todo lo elevada que se supone a los $36 \mathrm{~cm}$, teniendo en cuenta la tendencia de esta temperatura. Por otra parte se debe señalar que el nivel de los $36 \mathrm{~cm}$ está cerca del fondo de la paila de $40 \mathrm{~cm}$ de altura, zona que se puede considerar menos caliente 


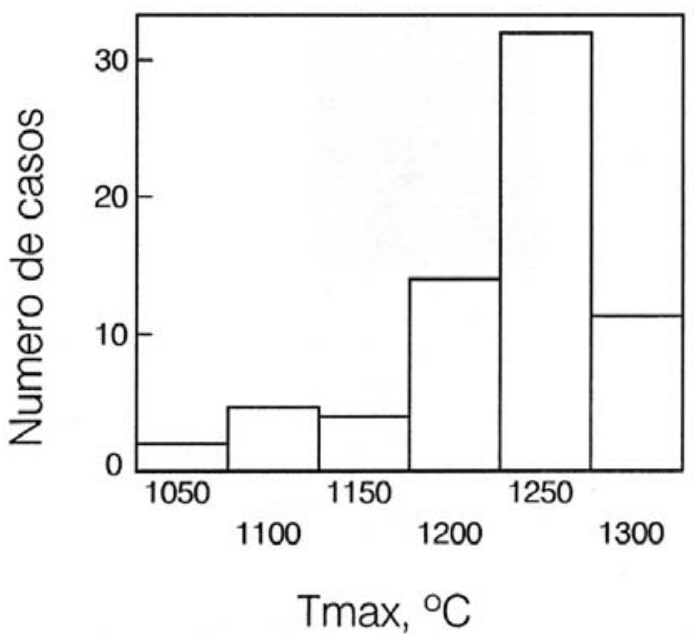

Figura 13. Histograma de las temperaturas máximas.

Figure 13. Maxim temperatures histograma.

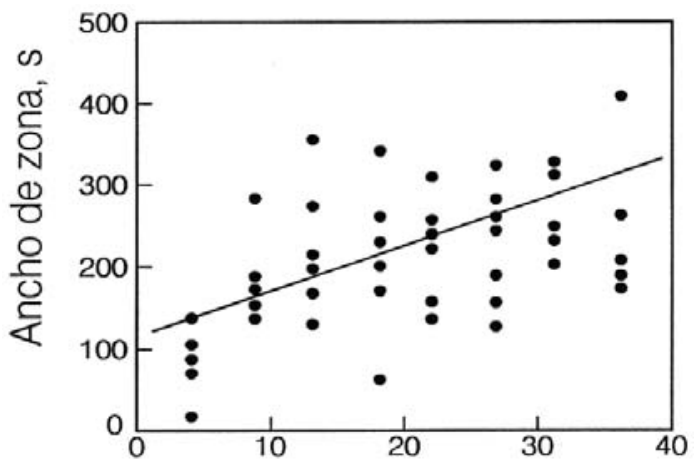

Profundidad del lecho, $\mathrm{cm}$

Figura 14. Evolución del ancho de la zona de sinterización con la profundidad del lecho.

Figure 14. Wide of the sintering zone evolution with the depth bed.

\section{CONCLUSIONES}

El trabajo realizado en una planta piloto de sinterización, con una paila alimentada con $110 \mathrm{~kg}$ de mezcla mineral, donde se han introducido una serie de termopares, a profundidades diferentes, permite establecer las siguientes conclusiones:

- Para la medida de temperaturas en el lecho de sinterización, se deben utilizar termopares de Platino/Platino-Rodio con una vaina de alúmina porosa para proteger el termopar y permitir una medida fiable de la temperatura, eliminando los efectos de inercia de las vainas no porosas.

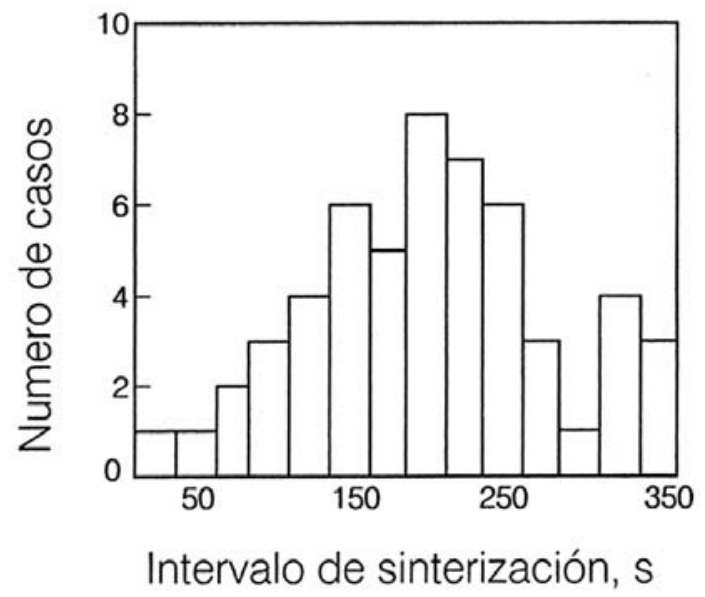

Figura 15. Histograma del ancho de zona de sinterización.

Figure 15. Wide of the sintering zone histogram.

- Los termopares se deben introducir en 8 puntos de medida, a alturas diferentes del lecho, para observar con fiabilidad la evolución del frente de llama a través del lecho. El período de muestreo será de $1 \mathrm{~s}$, ya que las velocidades de calentamiento y enfriamiento en el lecho son muy elevadas.

- El ancho de la zona de combustión y la temperatura máxima del frente de llama aumentan según aumenta la profundidad del lecho. El ancho de la zona de combustión se puede fijar en el intervalo comprendido entre los 50 y $350 \mathrm{~s}$. El valor medio de la Tmax se establece en el intervalo de $1.190-1.290^{\circ} \mathrm{C}$.

- La anomalía térmica observada en algunos ensayos, en la zona de $25-30 \mathrm{~cm}$, puede ser el resultado de un fenómeno de "mismatching" (desfase), provocado por una aspiración elevada y un régimen térmico confinado.

\section{REFERENCIAS}

[1] A. Moro, Tesis Doctoral, Escuela Técnica Superior de Ingenieros de Minas, Universidad de Oviedo, 1995.

[2] S.N.Ahsan, T.Mukherjee y J.A. Whiteman, Ironmaking Steelmaking 10 (1983) 54-64.

[3] T. Haga, A. Oshio, D. Shibata, S. Kasama y Y. Hida, Proc. $4^{\text {th }}$ European Cokemaking and Ironmaking Cong., vol. 1, París, Francia, Ed. ATS, París, Francia, 2000, pp. 118-125.

[4] A. Formoso, A. Moro, G. Fernandez-Pello, M. Muñiz, J. Jiménez, A. Moro y A. Cores, Rev. Metal. Madrid 36 (2000) 244-265. 
[5] A. Formoso, A. Moro, G. Fernandez-Pello, J.L. Menéndez, M. Muñíz y A. Cores, Ironmaking Steelmaking 30 (2003) 447-460.

[6] W. Davies, Can. Min. Metall. Bull. 63 (1960) 173-185.

[7] H. Toda, T. Senzaki, S. Isozaki y K. Kato, Trans. Iron Steel Inst. Jpn. 24 (1984) 187-196.

[8] Y. Ishikawa, M. Kase, M. Sasaki, K. Satoh y S. Sasaki, $41^{\text {th }}$ Ironmaking Conf. ISS, Pittsburg, EE. UU., Ed. Iron and Steel Soc. of Aime, 410 Commonwealth, Warrendale, PA 15086, EE. UU., 1982, pp. 80-89.

[9] J. Kurhara, A. Fukuda, S. Tanaka y S. Nigo, $42^{\text {th }}$ Ironmaking Conf. ISS, Atlanta, EE. UU., Ed. Iron and Steel Soc. of AIME, 410 Commonwealth, Warrendale, PA 15086, EE. UU., 1983, pp. 71-78.

[10] S. Yasumoto y S. Tanaka, Kawasaki Steel Tech. Rep. 5 (1982) 1-8.

[11] P. R. Dawson, Proc. $4^{\text {th }}$ Int. Symp. on Agglomeration, Toronto, Canadá, Ed. C. E. Capes, Iron and Steel Soc. of AIME, 410 Commonwealth, Warrendale, PA 15086, EE. UU., 1985, pp. 243-250.

[12] P. R. Dawson, J. Ostwald y K. M. Hayes, Proc. Australasia Inst. Min. Metall. 289 (1984) 163. 169.
[13] R. Nakajima, S. Kurosawa, H. Fukuyo y Y. Yamaoka, Proc. $6^{\text {th }}$ Int. Iron and Steel Cong., vol. 2, Nagoya, Japón, Ed. Iron and Steel Inst. of Japan, Tokyo, Japón, 1990, pp. 163-170.

[14] E. Marlière, C. Dulcy, C. Huguet y O. Leblanc, Proc. $3^{\text {rd }}$ European Cokemaking and Ironmaking Cong., vol. 1, Gent, Bélgica, Ed. CRM, Liège, Bélgica, 1996, pp. 202-208.

[15] W. Yang, C. Ryu, S.Choi, D. Lee y W. Huh, ISIJ Int. 44 (2004) 492-499.

[16] J. Mitterlehner, G. Loeffler, F. Winter, H. Hofbauer, H. Schmid, E. Zwittag, T. H. Buergler, O. Pammer y H. Stiasny, ISIJ Int. 44 (2004)11-20.

[17] Y. Ishikawa, Y. Shimomura, M. Sasaki, Y. Hida y H. Toda, $42^{\text {th }}$ Ironmaking Conf. ISS, Atlanta, EE. UU., Ed. Iron and Steel Soc. of AIME, 410, Commonwealth, Warrendale, PA 15086, EE. UU., 1983, pp. 17-29.

[18] F. Matsuno y T. Harada, Trans. Iron Steel Inst. Jpn. 21 (1981) 318-325.

[19] Li.-H. Hsieh y J. A. Whiteman, ISIJ Int. 29 (1989) 625-634.

[20] Li.-H. Hsieh y J.A. Whiteman, ISIJ Int. 29 (1989) 24-32.

[21] A. Cores, A. Babich, M. Muñiz, A. Isidro, S. Ferreira y R. Martin, Ironmaking Steelmaking 34 (2007) 231-240. 